\title{
Metrología y modulación en las mezquitas: propuesta de análisis a través de cuatro casos cordobeses*
}

\section{Metrology and modulation in mosques: an approach through four cases from Córdoba}

\author{
Carmen González Gutiérrez ${ }^{1}$ \\ Universidad de Córdoba
}

\section{RESUMEN}

En este artículo proponemos un acercamiento a la estructura y diseño arquitectónico de las mezquitas cordobesas mediante el análisis metrológico y de modulación de algunos conjuntos excavados en la capital andalusí. A través del estudio minucioso de las dimensiones de los patios y salas de oración, tratamos de determinar qué unidades métricas se aplicaron en el diseño y posterior ejecución de estos edificios, cómo se planteó su construcción o si existieron proporciones generales que rigiesen el diseño de cada una de sus partes. La aplicación de esta metodología a los conjuntos documentados ha permitido reconocer una tipología para las mezquitas califales cordobesas. Esta confirma la cronología de determinados conjuntos y sugiere detalles relativos a su organización interna, se relaciona con los ritmos constructivos detectados en la mezquita mayor, y permite hipótesis de reconstrucción de algunos conjuntos no documentados en su totalidad.

Palabras clave: mezquitas; Córdoba; metrología; codos; pie; arquitectura islámica.

\begin{abstract}
In this paper we suggest an approach to the structure and architectural design of mosques in Córdoba through the metrological and modulation analysis of some of them, excavated in the former capital of alAndalus. Through a careful study of courtyards and prayer rooms' dimensions, we sought to determine the metric units applied in the design and execution of these buildings, how they were constructed, or if there were general proportions that regulated the design of each of its parts. The application of this methodology to specific examples has allowed us to recognize a typology for caliphal mosques in Córdoba, which confirms the chronology of certain buildings and suggests details regarding their internal organization. It is also related to the constructive rhythms detected in the major mosque, and allows hypotheses of reconstruction of some complexes that were not fully excavated.
\end{abstract}

Key words: mosques; Córdoba; metrology; cubit; foot; Islamic architecture.

Recibido: 01-06-2017. Aceptado: 25-08-2017. Publicado online: 19-12-2017

Cómo citar este artículo / Citation

González Gutiérrez, C. 2017: "Metrología y modulación en las mezquitas: propuesta de análisis a través de cuatro casos cordobeses", Arqueología de la Arquitectura, 14: e058, doi: http://dx.doi.org/10.3989/arq.arqt.2017.018.

\section{Copyright}

(C) 2017 CSIC. Este es un artículo de acceso abierto distribuido bajo los términos de una licencia de uso y distribución Creative Commons Attribution (CC-by) España 3.0.

\footnotetext{
* El presente artículo recoge parte de los resultados obtenidos en el transcurso de la elaboración de nuestra Tesis Doctoral, titulada "Las mezquitas de la Córdoba islámica: concepto, tipología y función urbana”. Dirigida por el Prof. Dr. Desiderio Vaquerizo y el Prof. Dr. Alberto León, es el resultado de la concesión de una beca FPU del Ministerio de Educación y Ciencia de España, y fue defendida en enero de 2016 en la Universidad de Córdoba. Salvo que se indique lo contrario en su caso específico, todos los textos e imágenes contenidos en este artículo son obra de la autora.

1 carmengonz28@gmail.com / ORCID iD: http://orcid.org/0000-0003-0893-3932.
} 


\section{INTRODUCCIÓN Y OBJETIVOS}

A la hora de plantear el estudio arqueológico de las mezquitas secundarias de la Córdoba islámica, nos enfrentamos a una problemática muy concreta derivada de la falta de restos susceptibles de ser analizados con metodologías tradicionales. Hasta hoy, la arqueología tan solo ha logrado localizar apenas una quincena de conjuntos interpretables como mezquitas secundarias, cuyos estados de documentación y conservación son muy desiguales. Perviven algunos alminares, hoy transformados en campanarios de iglesias, pero las labores de restauración impiden, con demasiada frecuencia, un desarrollo adecuado de las lecturas paramentales. Por otra parte, la exhumación de las plantas completas de las mezquitas es del todo infrecuente y, en líneas generales, carecemos de información estratigráfica o planimétrica adecuada, que se agrava por la escasez de material arquitectónico, artístico u ornamental asociado a los restos. Dadas estas condiciones, la aplicación de las metodologías al uso para alcanzar una mejor comprensión arquitectónica de las mezquitas cordobesas resulta insuficiente, haciéndose necesaria la adopción de nuevos enfoques.

Las circunstancias descritas nos estimularon a proponer un acercamiento a la estructura y diseño arquitectónico de las mezquitas cordobesas a través del análisis de su modulación y metrología. El objetivo principal consiste en determinar cuál fue la unidad de medida empleada en el diseño de estos edificios. A raíz de ello, intentamos también aproximarnos a la forma en que fueron proyectados, así como a sus posteriores materializaciones físicas sobre el terreno. En segundo lugar, procuramos comprobar si hubo alguna relación proporcional entre las dimensiones de cada una de las partes estructurales de las mezquitas, o si existieron esquemas constructivos. De ser así, intentaremos la extrapolación de los patrones obtenidos a los casos documentados parcialmente, sugiriendo hipótesis sobre la ubicación y formas básicas de las partes del edificio que aún no se conocen.

Esta propuesta solamente puede probarse en ejemplos cuyas plantas se hayan excavado completas o lo más completas posible, con dimensiones escrupulosamente consignadas, y para los que se dispusiera de una planimetría fiable y precisa. Por ello, se ha aplicado tan solo en tres ejemplos cordobeses -las mezquitas de Fontanar, la Ronda Oeste y Santa Clara (vid. infra $)^{2}-$ y en uno proveniente de Madīnat al-Zahrā'.

\footnotetext{
2 Hemos elegido estos casos por ser los únicos cuya planta se ha documentado completa o casi completa. De los ejemplos cordobeses, dos se ubicaron extramuros, en los arrabales de occidentales -Fontanar y Ronda Oeste- y uno - Santa Clara- intramuros, cerca de la mezquita aljama.
}

\section{SISTEMA MÉTRICO Y UNIDADES DE MEDIDA EN AL-ANDALUS}

Los objetivos planteados requieren también de una aproximación al complejo universo del sistema de medidas que se utilizó en al-Andalus, determinante para el mundo de la construcción, y también uno de los pilares básicos y más eficaces de control de la economía del Estado (Jiménez Hernández 2015: 2). Sin embargo, mientras que el carácter universal del sistema métrico romano es un hecho asumido (Ibid.), no ocurre lo mismo con las unidades medievales, consideradas por muchos autores como "una amalgama de medidas de origen romano, visigodo y árabe a las que se añadirían las usadas por los constructores románicos y góticos, adaptadas además a las necesidades y circunstancias del lugar" (vid. Roldán 2013: 34). En el ámbito hispanomusulmán, además, los investigadores han percibido siempre una fuerte complicación del panorama debido al "empleo, bajo la común denominación de codos, de unidades que difieren bastante entre sí" (Hernández 1961-1962: 5) y que no en todos los casos respondieron a la cuantificación de medidas de longitud.

Hasta la fecha, numerosos investigadores han tratado, con desigual éxito, de identificar algunos de estos tipos de codo, así como su equivalencia en metros o centímetros actuales. De entre todos ellos, ${ }^{3}$ hay que subrayar la labor de F. Hernández (1961-1962), quien emprendió la titánica tarea de analizar la metrología de la mezquita aljama de Córdoba en sus múltiples fases, a través de la información aportada por las fuentes escritas y la realidad material. Tomando en consideración gran cantidad de variables, este autor concluyó que en el diseño y ejecución de la gran mezquita cordobesa se utilizaron, principalmente, dos tipos de codo: el denominado mammuní, para el que obtiene una equivalencia general de 47,14 $\mathrm{cm} ;{ }^{4}$ y el rassasí, que tuvo un valor de aproximadamente 58,93 cm (Hernández 1961-1962: 45). Con todo, no pudo determinar las razones que rigieron la elección de uno $u$ otro codo, pues no parecen observarse preferencias por ninguno de ellos a lo largo de los distintos siglos.

\footnotetext{
Otro autor que se ha acercado con solvencia a la problemática del codo y a sus posibles equivalencias con el sistema métrico actual, es J. Vallvé, quien describe, para época califal, la existencia de un codo manual -también llamado geométrico, antiguo o común- de $42 \mathrm{~cm}$; y otro rassasí de aproximadamente $58 \mathrm{~cm}$ que, según el autor, fue el oficial de la España musulmana (Vallvé 1976: 345-347). Los compendios de Vallvé ponen de manifiesto la enorme confusión que existe, al menos en la historiografía, en torno al término codo y a su equivalencia en metros contemporáneos.

4 Este es el mismo codo que F. Arnold aplica, exitosamente, en el estudio de un singular complejo arquitectónico califal localizado en el Vial Norte de Córdoba (vid. Arnold 2009-2010).
} 
Este autor desarrolló también el estudio arquitectónico del alminar de 'Abd al-Raḥmān III, proponiendo la hipótesis de que las plantas de los alminares de las mezquitas aljamas andalusíes habrían podido guardar una proporción de 10 x 10 codos (Hernández 1975: 174). Dicha propuesta ha servido de base a numerosos investigadores para establecer un tipo de codo casi exclusivo para cada edificio estudiado. ${ }^{5}$ Así, se ha asumido progresivamente que el sistema métrico andalusí fue inexacto, equívoco y profundamente variable, y ha tenido lugar una multiplicación tanto de maneras de aproximarse al tema como de resultados obtenidos para el análisis, en muchos casos, de un mismo edificio. ${ }^{6}$

A nuestro juicio, esta variación en la equivalencia del codo de unos edificios a otros carece de sentido. Puesto que "el patrón métrico afecta a todo el ciclo productivo, desde la modulación de los materiales de construcción, al aparejo constructivo y, con todo ello, al control de costos" (Jiménez Hernández 2015: 3), la equivalencia de sus unidades de medida no pudo deberse al azar o a criterios unipersonales, a la potestad de arquitectos o al capricho de gobernantes. Según un estudio reciente de A. Jiménez Hernández (2015: 3-6; vid. fig. 1), esta aparente multipli-

\footnotetext{
Tal y como queda recogido en la Tesis Doctoral de F. J. Roldán (2013 108), la aplicación de esta idea supondría que para la construcción del alminar de Madīnat al-Zahrā' se habría contemplado un codo de entre 52 y $53 \mathrm{~cm}$; de 51,5 para el alminar de la aljama zaragozana; o uno de entre 52 y $54 \mathrm{~cm}$ para el alminar de Tudela, entre otros ejemplos.

${ }^{6}$ La mezquita de Córdoba ha sido en numerosas ocasiones el leitmotiv de estudios de métrica, módulo y proporción, aunque éstos pocas veces han alcanzado conclusiones similares (vid., entre otros, Camps 1953; Hernández 1961-1962; De la Hoz 1973; Fernández Puertas 2000, 2008 y 2009 o Roldán 2013).
}

cidad de medidas se debió a la existencia de variaciones y submúltiplos que, extraídos de un mismo sistema métrico vigente, no supusieron nunca la modificación del mismo. La comprobación de esta idea en muy variados ejemplos arquitectónicos ${ }^{7}$ ha permitido a este autor identificar un sistema métrico válido en todo el mundo islámico medieval, basado en un pie de $31,43 \mathrm{~cm}$.

Según estos trabajos, este sistema guarda equivalencias exactas con otros anteriores y coetáneos a él, que Jiménez recoge en distintas tablas. Así, el codo mammuní $(47,14 \mathrm{~cm})$ habría equivalido a un pie y medio $(31,43+$ $15,715=47,145 \mathrm{~cm})$; y el rassasi $(58,93 \mathrm{~cm})$ a un pie y siete octavos $(31,43+27,50=58,93 \mathrm{~cm})$. Estas unidades de medida también tuvieron su equivalencia en dedos: 24 y 30 dedos respectivamente, midiendo el dedo $1,96 \mathrm{~cm}$. En resumen, el codo mammuní fue un codo común, de 24 dedos o 6 palmos menores, y el rassasí correspondió a un codo mayor de 30 dedos o 7,5 palmos (vid. Jiménez Hernández 2015: 4, fig. 2). Este último codo, además, era "una medida que establecía una equivalencia clara con el sistema romano, equivaliendo a dos pies romanos con precisión de un decimal" (Jiménez Hernández 2015: 4). ${ }^{8}$

Por tanto, nuestro análisis se desarrolla a partir de la afirmación de que el planteamiento y posterior

\footnotetext{
Jiménez Hernández ha aplicado con éxito esta propuesta de análisis a numerosos edificios islámicos y andalusíes, entre los que destacan una pequeña muestra de mezquitas, como la de la Cúpula de la Roca, la de Amman, las de Madīnat al-Zahrā', Sevilla o Tinmal (vid. Jiménez Hernández 2015: 18 y ss.), que le permiten afirmar que este sistema métrico no sólo tuvo validez en todo el territorio andalusí, sino que fue común a todo el Islam (Ibid.: 3 ).

8 Según este autor, muchos de los codos obtenidos por otros investigadores (vid. Roldán 2013: 118) son perfectamente convertibles a este sistema.
}

\begin{tabular}{|l|c|c|c|c|c|c|c|c|c|c|c|c|}
\hline & Dedo & Pulgada & Palmo & Palma & Pie & Palmipié & Codo & $\begin{array}{c}\text { Codo } \\
\text { 7 palmos }\end{array}$ & $\begin{array}{c}\text { Codo } \\
\mathbf{7} \text { palmos }\end{array}$ & $\begin{array}{c}\text { Codo } \\
\text { 2 pies }\end{array}$ & $\mathbf{2 , 5}$ pies & Vara \\
\hline & digitus & uncia & palmus & $\begin{array}{c}\text { palmus } \\
\text { maior }\end{array}$ & pes & palmipes & cubitus & & & & gradus & \\
\hline Drusiano & 2,08 & 2,78 & 8,33 & 25 & 33,33 & 41,66 & 50 & 58,33 & 62,49 & 66,66 & 83,33 & 99,99 \\
\hline & assba & & qabda & sibr & & & ma'muniyya & & rassasiyya & arsh & & qasab \\
\hline Islámico & 1,96 & 2,62 & 7,86 & 23,57 & 31,43 & 39,28 & 47,14 & 55 & 58,93 & 62,85 & 78,57 & 94,28 \\
\hline & digitus & uncia & palmus & $\begin{array}{c}\text { palmus } \\
\text { maior }\end{array}$ & pes & palmipes & cubitus & & & & gradus & \\
\hline Romano & 1,85 & 2,46 & 7,39 & 22,18 & 29,57 & 36,96 & 44,36 & 51,75 & 55,44 & 59,14 & 73,93 & 88,71 \\
\hline & & & & & & & & & & & & \\
\hline Castellano & 1,74 & 2,32 & 6,97 & 20,9 & 27,86 & 34,83 & 41,79 & 48,755 & 52,24 & 55,72 & 69,65 & 83,58 \\
\hline
\end{tabular}

Fig. 1: equivalencias entre las unidades (en centímetros) de los distintos sistemas métricos utilizados en Sevilla y su entorno durante la Edad Media, basadas en el pie. Así, un pie siempre equivale a dieciséis dedos, doce pulgadas o cuatro palmos menores (Jiménez Hernández 2015: 4, fig. 1). 
construcción de los edificios están fundamentados sobre relaciones concretas que, deriven o no en la existencia de ritmos constructivos, gozan de un módulo que no es caprichoso. Éste suele emplear relaciones numéricas enteras, y "se hace siempre sobre las anchuras básicas del trazado" (Camps 1953: 15-16). Así, trataremos de determinar a partir de qué proyecto se plantearon las mezquitas que hemos seleccionado y, posteriormente, cómo se trasladó dicho proyecto al terreno físico y con qué unidades. Para ello contemplaremos especialmente las medidas perimetrales exteriores de cada ejemplo ${ }^{9} \mathrm{y}$ tomaremos en mayor consideración las dimensiones de aquellos muros o crujías básicas y representativas para la estructuración y planeamiento del edificio. Siguiendo un criterio similar, primaremos la selección de muros de carga o crujías que definan o delimiten espacios, por encima de elementos decorativos, accesorios o secundarios, en nuestra creencia de que primero se diseñaron los elementos principales, y a partir de ellos se determinó lo secundario.

\section{METODOLOGÍA Y CASOS ESTUDIADOS}

Hemos confeccionado varias tablas para cada una de las mezquitas seleccionadas para nuestro estudio. Cada tabla recoge las dimensiones en metros de un espacio estructural (sala de oración o patio), así como su equivalencia en pies y en los codos identificados por F. Hernández en la aljama cordobesa (vid. supra). Debido a la posible deformación de las estructuras y a su estado de arrasamiento, a posibles imprecisiones de los levantamientos planimétricos, al desigual grado de documentación, etcétera, contemplamos el redondeo de las cifras obtenidas, pero nunca en más de un centímetro. Este criterio, aunque quizás demasiado ajustado si tenemos en cuenta el mal estado de conservación de los restos arqueológicos sobre los que trabajamos, nos parece esencial para asegurar la objetividad de nuestro trabajo, así como para evitar posibles deformaciones de los parámetros a nuestra conveniencia. Por último, valoramos la obtención de medidas lo más íntegras posibles -enteras y medias-, en la línea de lo postulado por Camps (1953) y Jiménez Hernández (2015). En las citadas tablas sombreamos aquellas cifras que indican

\footnotetext{
9 En el estudio de la mezquita de Córdoba, por ejemplo, F. Hernández descubre que las menciones que los autores árabes hacen a las medidas corresponden prácticamente en todos los casos a las medidas exteriores. Éstas suelen contemplar la inclusión de los elementos que sobresalen de la mezquita, como por ejemplo los contrafuertes, aunque no siempre el mihrab.
}

codos enteros o medios codos, pies enteros o medios pies, pues son las que, a la luz de las lecturas previamente citadas, consideramos que pudieron emplearse para la proyección sobre el terreno de estos edificios.

\section{Caso 1: mezquita de Fontanar}

Según los datos extraídos de la excavación arqueológica en la que se documentó (vid. Luna y Zamorano 1999; González Gutiérrez 2016a: 109 y ss.), este conjunto tuvo una única fase constructiva, en época califal, sin que experimentara modificaciones ni remodelaciones sustanciales a lo largo de su vida útil. Pero, aunque su construcción sobre el terreno se realizase en un mismo momento, para aproximarnos a cómo se pudo plantear su diseño sobre el plano analizaremos cada espacio por separado:

SALA DE ORACIÓN: su forma prácticamente cuadrada induce a pensar que su diseño se planteó a partir del trazado de un círculo, en el que dicho cuadrado habría quedado inscrito posteriormente (Fig. 2). Las dimensiones de estas figuras geométricas, correspondientes al posible diseño del arquitecto en el plano, así como las propias de los restos arqueológicos documentados, derivadas de la ejecución de la obra, quedan resumidas en la tabla siguiente (Fig. 3).

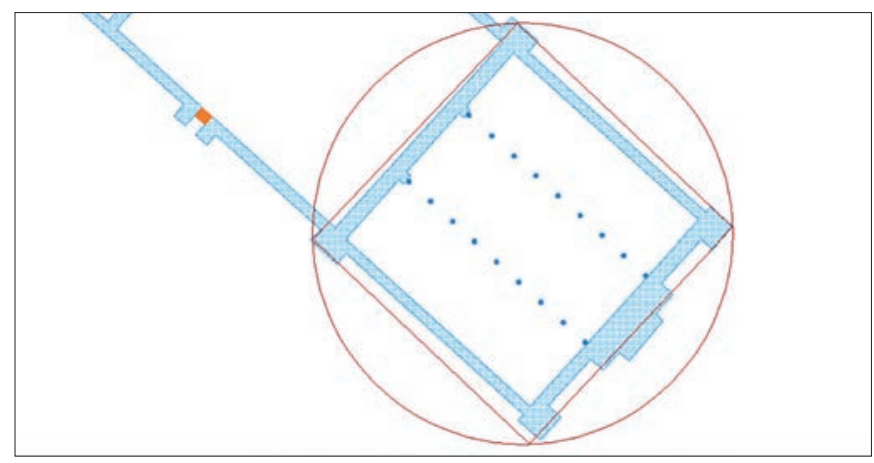

Fig. 2: hipótesis del trazado de la sala de oración de la mezquita de Fontanar.

\begin{tabular}{|c|c|c|c|c|}
\hline $\begin{array}{c}\text { FONTANAR: } \\
\text { SALA DE } \\
\text { ORACIÓN }\end{array}$ & Metros & $\begin{array}{c}\text { Codos } \\
\text { rassasís } \\
(0,5893)\end{array}$ & $\begin{array}{c}\text { Codos } \\
\text { mammunís } \\
(0,4714)\end{array}$ & $\begin{array}{c}\text { Pies } \\
(0,3143)\end{array}$ \\
\hline Radio círculo & 15,54 & 26,37 & $32,96=33$ & $49,45=49,5$ \\
\hline $\begin{array}{c}\text { Lado del cuadrado } \\
\text { inscrito }\end{array}$ & 22 & 37,33 & 46,67 & $69,99=70$ \\
\hline $\begin{array}{c}\text { Diagonal del } \\
\text { cuadrado inscrito }\end{array}$ & 31,11 & $74,07=74$ & $65,99=66$ & $98,98=99$ \\
\hline Anchura exterior & 21,22 & 36 & 45 & 67,5 \\
\hline Longitud exterior & 21,72 & 36,85 & $46,07=46$ & $69,1=69$ \\
\hline
\end{tabular}

Fig. 3: dimensiones de la sala de oración de Fontanar. 
Como podemos observar, tanto nuestra hipótesis de trazado como la posterior plasmación material de la misma - los restos documentados- se corresponden con medidas en pies, lo cual nos lleva a pensar que dicha hipótesis puede ser correcta. Ello significaría que esta sala o haram se concibió como un cuadrado de 70 pies de lado que, al ser trasladado al terreno, quedó como un espacio cuadrangular de $67,5 \times 69$ pies.

PATIO: el patio de esta mezquita también tiene forma cuadrangular, aunque no tan exacta como la sala de oraciones. Por dicho motivo, hemos planteado el análisis de sus dimensiones de la misma manera que para el espacio anterior, con el que comparte anchura. Consideramos dos longitudes distintas, una con pórtico y otra sin él (figs. 4 y 5). En claro contraste con los resultados obtenidos en el análisis del espacio anterior, en esta ocasión no parece que esta área se trazase de la forma en que proponemos.

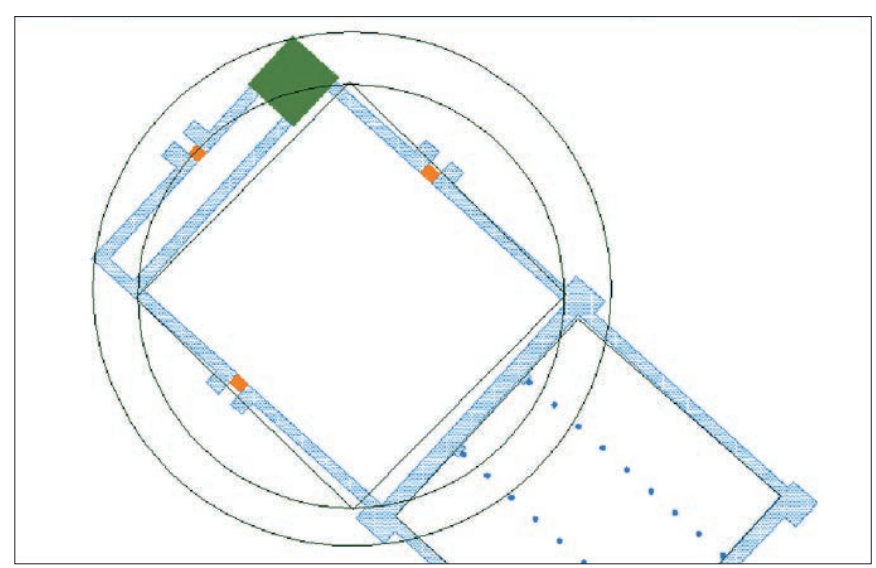

Fig. 4: hipótesis del trazado del patio de Fontanar.

\begin{tabular}{|c|c|c|c|c|}
\hline $\begin{array}{c}\text { FONTANAR: } \\
\text { PATIO }\end{array}$ & Metros & $\begin{array}{c}\text { Codos } \\
\text { rassasís } \\
(0,5893)\end{array}$ & $\begin{array}{c}\text { Codos } \\
\text { mammunís } \\
(0,4714)\end{array}$ & $\begin{array}{c}\text { Pies } \\
(0,3143)\end{array}$ \\
\hline Anchura exterior & 21,22 & 36 & 45 & 67,5 \\
\hline $\begin{array}{c}\text { Longitud exterior sin } \\
\text { pórtico }\end{array}$ & 21,86 & 37 & 43,37 & 69,5 \\
\hline $\begin{array}{c}\text { Radio círculo sin } \\
\text { pórtico }\end{array}$ & 14,40 & $\begin{array}{c}24,43= \\
24,5\end{array}$ & 30,5 & 45,80 \\
\hline $\begin{array}{c}\text { Lado del cuadrado } \\
\text { inscrito sin pórtico }\end{array}$ & 20,547 & 34,87 & $43,58=43,5$ & 65,37 \\
\hline $\begin{array}{c}\text { Diagonal del } \\
\text { cuadradoinscrito sin } \\
\text { pórtico }\end{array}$ & 29,05 & 49,29 & 61,62 & $90,42=92,5$ \\
\hline Longitud pórtico & 4,25 & 7,21 & $9,01=9$ & $13,52=13,5$ \\
\hline $\begin{array}{c}\text { Longitud patio } \\
\text { completo }\end{array}$ & 25,82 & 43,84 & 54,77 & 82,22 \\
\hline $\begin{array}{c}\text { Radio círculo con } \\
\text { pórtico }\end{array}$ & 17,49 & 29,68 & 37 & 55,64 \\
\hline
\end{tabular}

Fig. 5: dimensiones del patio de Fontanar.
MEZQUITA TOTAL: a simple vista, los resultados relacionados con el patio no son concluyentes, por lo que hemos elaborado también una tabla (Fig. 6) en la que se resumen las dimensiones globales de esta mezquita, para comprobar si la adición del patio al haram guardó alguna relación proporcional con éste.

\begin{tabular}{|c|c|c|c|c|}
\hline $\begin{array}{c}\text { FONTANAR. } \\
\text { MEZQUITA } \\
\text { COMPLETA }\end{array}$ & Metros & $\begin{array}{c}\text { Codos } \\
\text { rassasis } \\
(0,5893)\end{array}$ & $\begin{array}{c}\text { Codos } \\
\text { mammunis } \\
(0,4714)\end{array}$ & $\begin{array}{c}\text { Pies } \\
(0,3143)\end{array}$ \\
\hline $\begin{array}{c}\text { Anchura } \\
\text { exterior }\end{array}$ & 21,22 & 36 & 45 & $67,51=67,5$ \\
\hline $\begin{array}{c}\text { Longitud } \\
\text { exterior sin } \\
\text { pórtico }\end{array}$ & 43,56 & $73,91=74$ & 92,40 & $138,59=138,50$ \\
\hline Longitud total & 47,50 & $\begin{array}{c}80,59= \\
80,50\end{array}$ & 100,74 & $151,1=151$ \\
\hline
\end{tabular}

Fig. 6: dimensiones de la mezquita de Fontanar analizada en su conjunto.

A la luz de estos datos, interpretamos que la sala de oración es el primer espacio que se plantea, a partir de un círculo y un cuadrado inscrito en él de 70 pies de lado. La ejecución de la obra supuso una mínima modificación de esas medidas, lo cual llevó a esta sala a tener 67,5 pies de ancho por 69 de largo. A ella parece añadirse, sin influir en su diseño, el patio que, sin pórtico, tiene unas dimensiones prácticamente idénticas al haram (67,5 pies de ancho $\mathrm{x}$ 69,5 de largo). La adición del pórtico, pese a no deberse a una reforma cronológica posterior, rompe esa simetría. No obstante, las dimensiones totales y finales de la mezquita se pueden medir en pies, como se observa en la figura 6 .

Al margen de la simetría casi exacta entre la sala de oración y el patio sin pórtico, no hemos encontrado en este ejemplo ninguna razón proporcional que justifique la elección de dichas medidas, como sí ocurre en otros casos. ${ }^{10}$ Sin embargo, resulta interesante la comparación, a grandes rasgos, entre las formas de la mezquita de Fontanar y la planimetría, en sus distintas fases de crecimiento, de la Gran Mezquita, cuyo resultado confirma la cronología califal del conjunto que estamos analizando. La mezquita de los viernes tuvo, en origen, una planta total cuadrada, en la que oratorio y patio contaron aproximadamente con la misma superficie. Este equilibrio fue alterado con la construcción, en tiempos de Hišām I, de un alminar que se proyectaba hacia el exterior. La ampliación hacia el sur de 'Abd al-Raḥmān II no devolvió al edificio esa armonía en

10 Los análisis de A. Jiménez Hernández demuestran cómo la anchura de algunas salas de oración respondió a la multiplicación de la longitud total por $\sqrt{ } 2$ (Jiménez Hernández 2015: 21 y ss.). 
sus proporciones, la cual no se recuperaría hasta el añadido de 'Abd al-Raḥmān III. Con él, patio y sala de oraciones volvieron a tener, a grandes rasgos, la misma área. La ruptura definitiva de este equilibrio se produciría con la última ampliación hacia el mediodía, ordenada por al-Ḥakam II (Hernández 1961-1962: 40).

En la mezquita que nos ocupa se observa una proporción casi exacta entre el espacio otorgado a la sala de oraciones y el destinado al patio, sin contar su pórtico. Por tanto, en comparación con la aljama, estamos ante un tipo sin duda alguna califal, bien de 'Abd al-Rạ̣mān III por el acusado equilibrio de superficie imperante entre la sala y el patio, bien de al-Hakam II por la ruptura de ese equilibrio con la adición del espacio porticado.

\section{Caso 2: mezquita de la Ronda Oeste}

Esta mezquita fue fechada en un primer momento en época califal por la arqueóloga responsable de su excavación, pero posteriormente se ha propuesto un origen emiral para la misma, con una posible fase de ampliación califal. ${ }^{11}$ Nuestro análisis pretende comprobar si la configuración final de esta mezquita se debió a la existencia de dos fases constructivas distintas, para lo cual analizamos los espacios de forma análoga al caso 1.

SALA DE ORACIÓN: en esta ocasión, las dimensiones exteriores no están determinadas por contrafuertes. No obstante, contamos con un mihrab que se proyecta hacia el exterior y que nos hace contemplar dos posibles longitudes, como reflejamos en las figs. 7 y 8 . Por otra parte, dada la forma rectangular de la sala, descartamos su trazado a partir de un cuadrado inscrito en un círculo.

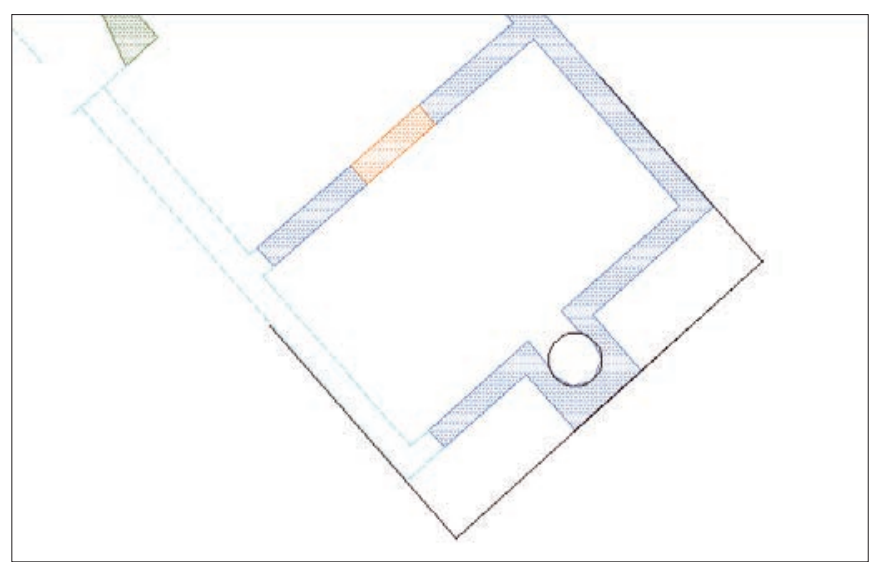

Fig. 7: propuesta del trazado de la sala de oración de la mezquita de la Ronda Oeste.

\footnotetext{
11 Los detalles de esta discusión están compilados en González Gutiérrez 2016a: 139.
}

\begin{tabular}{|c|c|c|c|c|}
\hline $\begin{array}{c}\text { RONDA OESTE. } \\
\text { SALA DE } \\
\text { ORACIÓN }\end{array}$ & Metros & $\begin{array}{c}\text { Codos } \\
\text { rassasís } \\
(0,5893)\end{array}$ & $\begin{array}{c}\text { Codos } \\
\text { mammunís } \\
(0,4714)\end{array}$ & $\begin{array}{c}\text { Pies } \\
(0,3143)\end{array}$ \\
\hline Ancho exterior & 8,94 & 15,17 & $18,96=19$ & $28,44=28,5$ \\
\hline $\begin{array}{c}\text { Longitud } \\
\text { exterior sin } \\
\text { mihrab }\end{array}$ & 5,95 & $10,09=10$ & 12,62 & $18,93=19$ \\
\hline $\begin{array}{c}\text { Longitud } \\
\text { exterior con } \\
\text { mihrab }\end{array}$ & 7,55 & 12,81 & $16,01=16$ & $24,04=24$ \\
\hline $\begin{array}{c}\text { Longitud mihrab } \\
\text { solo }\end{array}$ & 1,63 & 2,76 & $3,45=3,5$ & 5,18 \\
\hline $\begin{array}{c}\text { Círculo sala de } \\
\text { oración con } \\
\text { mihrab: radio }\end{array}$ & 5,84 & 10 & 12,39 & $18,58=18,50$ \\
\hline Mihrab: radio & 0,59 & 1 & 1,25 & 1,88 \\
\hline
\end{tabular}

Fig. 8: dimensiones de la sala de oración de Ronda Oeste.

A la luz de los datos contenidos en la figura 8, nos queda bastante clara la plasmación del proyecto de obra sobre el terreno: una sala de oración rectangular de 19 o 24 pies de longitud, dependiendo esto de si consideramos o no el mihrab, por 28,5 pies de anchura. Aunque sea evidente, una vez más, la utilización del pie en el planteamiento de las magnitudes lineales, no hemos logrado esclarecer cuál fue el proyecto inicial del arquitecto, que parece alejarse del esquema seguido en Fontanar. Además, encontramos una cuestión bastante llamativa en el trazado del mihrab, que dibuja un círculo al interior cuyo radio mide, exactamente, un codo rassasí.

PATIO: para el estudio del patio consideraremos dos modalidades: la versión sin pórtico (Figs. 9 y 10), que constituye según algunos investigadores la parte inicial de la construcción (Murillo, Casal y Castro 2004: 267); y una segunda con el pórtico ya añadido (Figs. 11 y 12).

a) Patio sin pórtico:

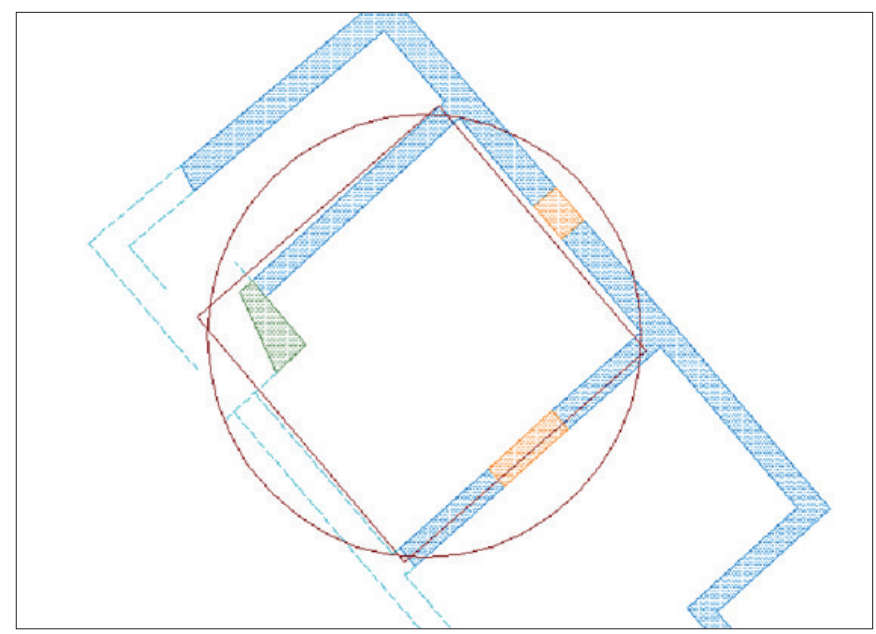

Fig. 9: vista del patio de Ronda Oeste con la hipótesis de su trazado interior, sin pórtico, a partir de un círculo. 


\begin{tabular}{|c|c|c|c|c|}
\hline $\begin{array}{c}\text { RONDA } \\
\text { OESTE. PATIO } \\
\text { SIN PÓRTICO }\end{array}$ & Metros & $\begin{array}{c}\text { Codos } \\
\text { rassasís } \\
(0,5893)\end{array}$ & $\begin{array}{c}\text { Codos } \\
\text { mammunis } \\
(0,4714)\end{array}$ & $\begin{array}{c}\text { Pies } \\
(0,3143)\end{array}$ \\
\hline $\begin{array}{c}\text { Anchura } \\
\text { exterior }\end{array}$ & 8,94 & 15,17 & $18,96=19$ & $28,44=28,5$ \\
\hline $\begin{array}{c}\text { Longitud } \\
\text { exterior }\end{array}$ & 6,30 & 10,70 & 13,36 & $20,04=20$ \\
\hline Radio circulo & 5,735 & 9,73 & 12,16 & 18,24 \\
\hline $\begin{array}{c}\text { Lado cuadrado } \\
\text { inscrito }\end{array}$ & 8,11 & 13,76 & 17,20 & 25,80 \\
\hline $\begin{array}{c}\text { Diagonal } \\
\text { cuadrado } \\
\text { inscrito }\end{array}$ & 11,47 & $19,46=19,50$ & 24,33 & $\begin{array}{c}36,49= \\
36,50\end{array}$ \\
\hline
\end{tabular}

Fig. 10: dimensiones del patio de Ronda Oeste sin su pórtico.

Tal y como ocurre con la sala de oraciones, se evidencia el uso del pie en la ejecución de la obra de este espacio (28,5 x 20 pies), aunque la correspondencia de la longitud con la anchura multiplicada por $\sqrt{2}$ ( $v i d$. nota 9) no se halla tampoco en este caso. La aplicación de este método de análisis no nos ha permitido, por tanto, conocer cómo se proyectó este patio sobre el papel.

b) Patio con pórtico:

Con la construcción del pórtico, el patio o sahn se convierte en un espacio mucho más cuadrangular $(8,94$ x 9,70 m). Con todo, el análisis de este espacio con su pórtico parece complicar más que esclarecer el panorama, pues no se observan equivalencias claras en las unidades de medida. Puesto que anchura y longitud del patio sin pórtico se pueden medir en pies, y se descuadran al añadir el espacio porticado, cabría suponer que éste no se contempló en el diseño inicial del sahn.

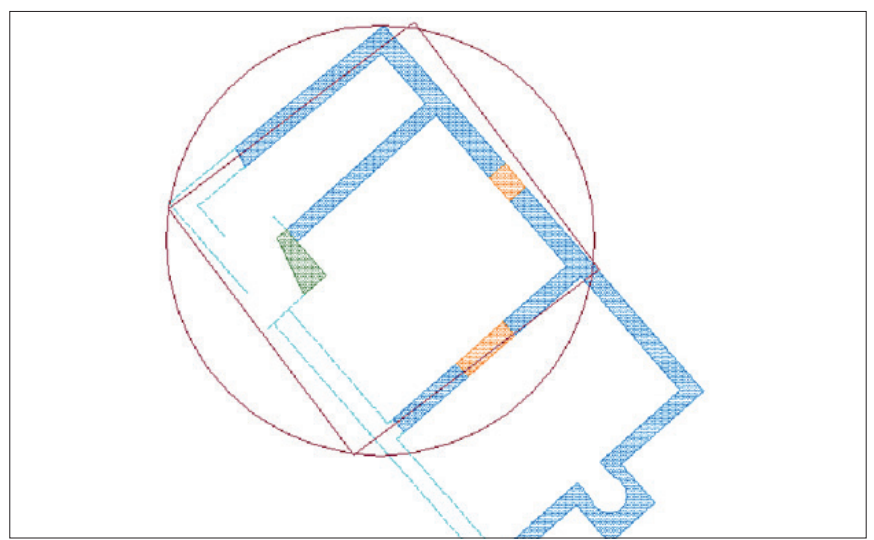

Fig. 11: vista del patio de Ronda Oeste con la hipótesis de su trazado, esta vez con pórtico, a partir de un círculo. Aunque no se ajusta exactamente a la ubicación del sahn, el cuadrado inscrito concuerda mucho mejor con las dimensiones del mismo, al contrario de lo que sucedía en el caso anterior.

\begin{tabular}{|c|c|c|c|c|}
\hline $\begin{array}{c}\text { RONDA } \\
\text { OESTE. PATIO } \\
\text { CON PÓRTICO }\end{array}$ & Metros & $\begin{array}{c}\text { Codos } \\
\text { rassasís } \\
(0,5893)\end{array}$ & $\begin{array}{c}\text { Codos } \\
\text { mammunís } \\
(0,4714)\end{array}$ & Pies (0,3143) \\
\hline $\begin{array}{c}\text { Anchura } \\
\text { exterior }\end{array}$ & 8,94 & 15,17 & $18,96=19$ & $28,44=28,5$ \\
\hline $\begin{array}{c}\text { Longitud } \\
\text { exterior pórtico }\end{array}$ & 3,40 & 5,77 & 7,21 & 10,81 \\
\hline $\begin{array}{c}\text { Longitud patio } \\
\text { completo }\end{array}$ & 9,63 & 16,34 & $20,42=20,50$ & 30,63 \\
\hline $\begin{array}{c}\text { Radio círculo } \\
\text { patio completo }\end{array}$ & 6,73 & $11,42=11,50$ & 14,27 & $21,41=21,5$ \\
\hline $\begin{array}{c}\text { Lado cuadrado } \\
\text { inscrito }\end{array}$ & 9,5 & 16,12 & 20,15 & 30,22 \\
\hline $\begin{array}{c}\text { Diagonal } \\
\text { cuadrado } \\
\text { inscrito }\end{array}$ & 13,435 & 22,80 & 28,5 & 42,74 \\
\hline
\end{tabular}

Fig. 12: dimensiones del patio de Ronda Oeste con el pórtico añadido.

MEZQUITA COMPLETA: como en el caso anterior, hemos completado este análisis con la consideración de las dimensiones de la mezquita como un todo, en primer lugar sin pórtico (Fig. 13), y posteriormente con él (Fig. 14).

\begin{tabular}{|c|c|c|c|c|}
\hline $\begin{array}{c}\text { RONDA OESTE. } \\
\text { MEZQUITA COMPLETA } \\
\text { SIN PORTICO }\end{array}$ & Metros & $\begin{array}{c}\text { Codos rassasís } \\
(0,5893)\end{array}$ & $\begin{array}{c}\text { Codos } \\
\text { mammunís } \\
(0,4714)\end{array}$ & Pies $(0,3143)$ \\
\hline Anchura exterior & 8,94 & 15,17 & $18,96=19$ & $28,44=28,5$ \\
\hline $\begin{array}{c}\text { Longitud exterior sin } \\
\text { milhrab }\end{array}$ & 12,25 & 20,78 & $25,98=26$ & $38,97=39$ \\
\hline $\begin{array}{c}\text { Longitud exterior con } \\
\text { mihrab }\end{array}$ & 14,25 & 24,18 & 30,23 & 45,33 \\
\hline
\end{tabular}

Fig. 13: dimensiones completas de la mezquita de la Ronda Oeste, sin pórtico.

\begin{tabular}{|c|c|c|c|c|}
\hline $\begin{array}{c}\text { RONDA OESTE. } \\
\text { MEZQUITA COMPLETA } \\
\text { CON PORTICO }\end{array}$ & Metros & $\begin{array}{c}\text { Codos } \\
\text { rassasis } \\
(0,5893)\end{array}$ & $\begin{array}{c}\text { Codos } \\
\text { mammunis } \\
(0,4714)\end{array}$ & Pies (0,3143) \\
\hline Anchura exterior & 8,94 & 15,17 & $18,96-19$ & $28,44=28,5$ \\
\hline Longitud exterior sin mihrab & 15,54 & 26,37 & $32,96-33$ & $49,44=49,50$ \\
\hline Longitud exterior total & 17,25 & 29,27 & 36,59 & 54,88 \\
\hline
\end{tabular}

Fig. 14: dimensiones completas de la mezquita de la Ronda Oeste, con pórtico.

Los resultados arrojados por estas figuras no nos permiten discernir si la mezquita se planteó desde un primer momento con pórtico o sin él pues, en ambos casos, las magnitudes lineales coinciden tanto con pies como con codos mammuníes. La contabilización del mihrab supone un descuadre en la longitud total para la que creemos poder ofrecer una explicación: es posible que la sala de oración no se proyectase desde fuera hacia adentro, como veníamos considerando, sino al revés. De ser así, el arquitecto no habría primado en sus cálculos, la superficie del espacio disponible -lo cual no resulta descabellado ya que estamos hablando de un área libre de construcciones en aquel momento y, por tanto, sin restricciones urbanísticas-, sino que habría tenido en cuenta, por encima de todo, la superficie necesaria para conseguir el aforo deseado al interior del 
haram. De resultar cierta esta propuesta, habría que contabilizar tan sólo las medidas interiores y excluir al mihrab, de la siguiente manera (Fig. 15):

\begin{tabular}{|c|c|c|c|c|}
\hline $\begin{array}{c}\text { RONDA OESTE. } \\
\text { SALA DE } \\
\text { ORACIÓN }\end{array}$ & Metros & $\begin{array}{c}\text { Codos } \\
\text { rassasís } \\
(0,5893)\end{array}$ & $\begin{array}{c}\text { Codos } \\
\text { mammunis } \\
(0,4714)\end{array}$ & $\begin{array}{c}\text { Pies } \\
(0,3143)\end{array}$ \\
\hline Anchura interior & 7,70 & $13,06=13$ & 16,33 & $24,49=24,50$ \\
\hline $\begin{array}{c}\text { Longitud } \\
\text { interior }\end{array}$ & 4,85 & 8,32 & 10,29 & $15,43=15,5$ \\
\hline
\end{tabular}

Fig. 15: dimensiones interiores del haram de la Ronda Oeste.

Las magnitudes obtenidas, reflejadas en la figura 15 , apoyan esta propuesta, en la que de nuevo prima la utilización del pie por encima de cualquier otro múltiplo o submúltiplo. Por el contrario, la comparativa con la mezquita de los viernes no puede efectuarse tan claramente como para el caso 1. Aquí, haram y patio no guardan simetría, ni siquiera aproximada, en sus formas o superficies, pues nos encontramos ante una mezquita longitudinal que no sigue el modelo de la mezquita aljama primitiva. La preeminencia de la longitud por encima de la anchura sí se da a finales de época emiral en la gran mezquita cordobesa, pero con una notable predominancia del haram sobre el sahn, circunstancia que no observamos aquí, en la Ronda Oeste. La adición o no de un pórtico aquí nunca supuso la creación ni la recuperación de un equilibrio entre la superficie dedicada a ambos espacios, como sí ocurre en la aljama. Por tanto, a simple vista, la comparación de las formas de este oratorio con la gran mezquita no nos aporta ninguna pista sobre su posible cronología.

\section{Caso 3: mezquita menor de Madīnat al- Zahrā'}

Nos encontramos ante otra mezquita erigida en el Califato (vid. Vallejo 2009), bien durante el mandato de 'Abd al-Raḥmān III o bien de su sucesor, al-Hakam II. $\mathrm{Su}$ análisis por espacios se plasma así:

SALA DE ORACIÓN: como en los casos precedentes, hemos examinado en primer lugar las dimensiones exteriores del haram. El planteamiento de un trazado cuadrangular a partir de un círculo no parece tener sentido en esta ocasión, tal y como se desprende de la figura 16. Además, esta mezquita incluye una serie de elementos, tales como un contrafuerte ${ }^{12} \mathrm{o}$ un mihrab proyectado

\footnotetext{
12 La existencia de contrafuertes tan sólo en uno de los lados de la mezquita nos llevó a pensar que quizás esta falta de simetría se debiese a la adaptación del edificio al espacio existente, determinado por la posición de la muralla. Sin embargo, Vallejo afirma que la construcción de la mezquita fue anterior a la de la cerca muraria (Vallejo 2009: 221), por lo que desechamos esa hipótesis.
}

al exterior, que nos obligan a contemplar varias combinaciones (Fig. 17):

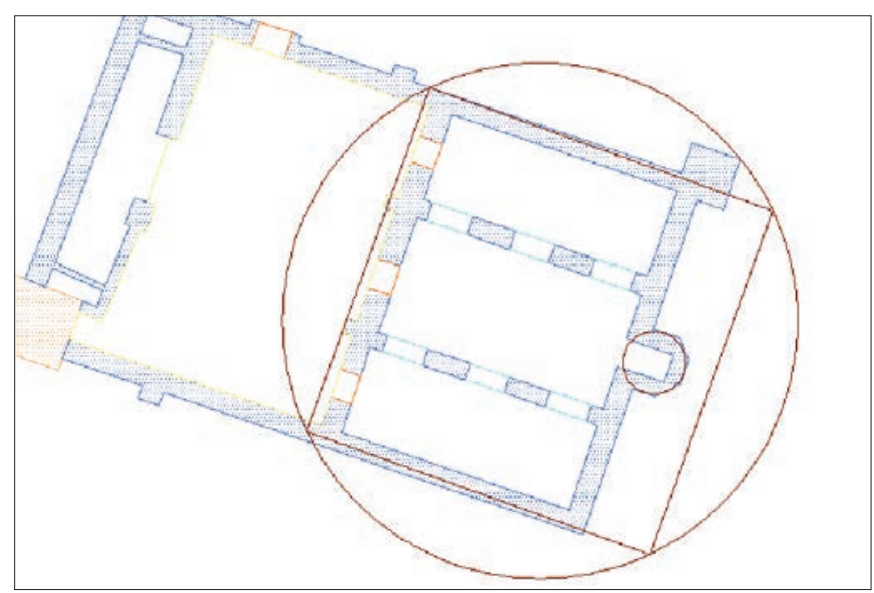

Fig. 16: propuesta de trazado de la sala de oración de la pequeña mezquita de Madīnat al-Zahrā'. Los límites del cuadrado propuesto no coinciden con los del haram en su lado meridional.

\begin{tabular}{|c|c|c|c|c|}
\hline $\begin{array}{c}\text { MEZQUITA DE } \\
\text { MADINAT AL- } \\
\text { ZAHRA'. } \\
\text { SALA DE ORACIÓN }\end{array}$ & Metros & $\begin{array}{c}\text { Codos } \\
\text { rassasís } \\
(0,5893)\end{array}$ & $\begin{array}{c}\text { Codos } \\
\text { mammunís } \\
(0,4714)\end{array}$ & $\begin{array}{c}\text { Pies } \\
(0,3143)\end{array}$ \\
\hline $\begin{array}{c}\text { Anchura exterior sin } \\
\text { elementos }\end{array}$ & 15,54 & 26,37 & $32,96=33$ & $\begin{array}{c}49,44= \\
49,50\end{array}$ \\
\hline $\begin{array}{c}\text { Anchura exterior con } \\
\text { elementos }\end{array}$ & 16,90 & 28,68 & 35,85 & 53,77 \\
\hline $\begin{array}{c}\text { Longitud exterior sin } \\
\text { elementos }\end{array}$ & 11,78 & $19,98=20$ & $24,98=25$ & $\begin{array}{c}37,48 \\
37,5\end{array}$ \\
\hline $\begin{array}{c}\text { Longitud exterior con } \\
\text { elementos }\end{array}$ & 12,96 & $21,99=22$ & $27,49=27,5$ & 41,23 \\
\hline Longitud mihrab & 1,82 & $3,08=3$ & $22,03=22$ & $33,04=$ \\
\hline Radio círculo & 10,72 & 18,19 & 22,74 & 34,10 \\
\hline Radio mihrab & 1,30 & 2,2 & 2,75 & 4,13 \\
\hline
\end{tabular}

Fig. 17: dimensiones exteriores del haram de la mezquita de Madīnat al-Zahrā'.

A tenor de estos datos podría deducirse que la proyección de este espacio se planteó en ausencia de sus elementos exteriores. El rectángulo que dibuja la sala de oración, de 49,5 × 37,5 pies, tampoco sigue la proporción de anchura $\mathrm{x} \sqrt{2}=$ longitud, ni a la inversa. Sin embargo, la longitud exterior sin elementos, multiplicada por $\sqrt{2}$, da un ancho de 53 pies, que sí se aproxima más a los 53,77 pies de la anchura con el contrafuerte.

PATIO: tampoco parece que este espacio se proyectase a partir del trazado de círculo-cuadrado que sí hemos podido comprobar en el caso 1 . El estudio de sus dimensiones tampoco parece ser más concluyente, como se aprecia en las figs. 18 y 19: 


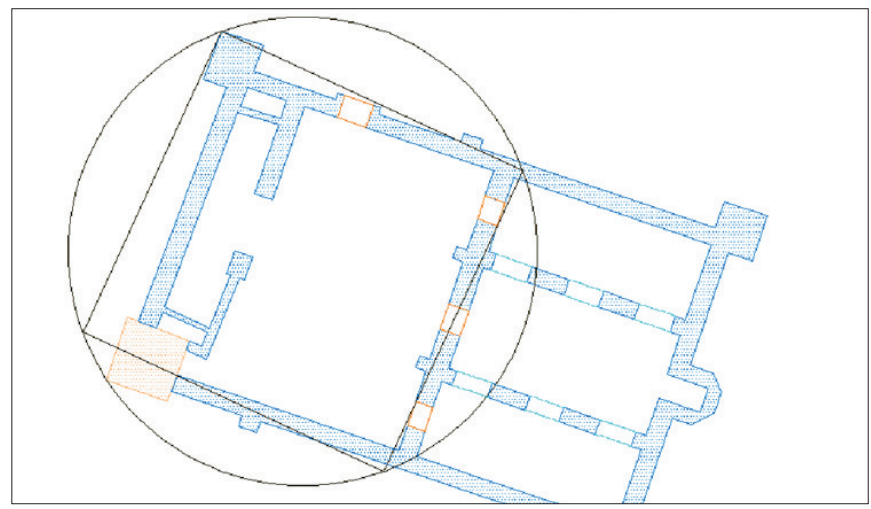

Fig. 18: propuesta del trazado del patio de esta mezquita. De nuevo, el cuadrado que se inserta en el círculo que alberga el área del patio no coincide con su perímetro básico.

\begin{tabular}{|c|c|c|c|c|}
\hline $\begin{array}{c}\text { MEZQUITA DE MADINNAT } \\
\text { AL-ZAHRA } \bar{A}^{\prime} \text {. } \\
\text { PATIO }\end{array}$ & Metros & $\begin{array}{c}\text { Codos } \\
\text { rassasis } \\
(0,5893)\end{array}$ & $\begin{array}{c}\text { Codos } \\
\text { mammunis } \\
(0,4714)\end{array}$ & $\begin{array}{c}\text { Pies } \\
(0,3143)\end{array}$ \\
\hline $\begin{array}{c}\text { Anchura exterior sin } \\
\text { contrafuertes }\end{array}$ & 15,54 & 26,37 & $32,96=33$ & $\begin{array}{c}49,44= \\
49,50\end{array}$ \\
\hline Longitud exterior sin pórtico & 10,36 & $\begin{array}{c}17,58= \\
17,50\end{array}$ & $21,98=22$ & $32,96=33$ \\
\hline $\begin{array}{c}\text { Anchura exterior con } \\
\text { contrafuertes }\end{array}$ & 17,41 & $\begin{array}{c}29,54= \\
29,50\end{array}$ & $36,93=37$ & $\begin{array}{c}29,54= \\
29,50\end{array}$ \\
\hline $\begin{array}{c}\text { Longitud exterior con pórtico } \\
\text { y contrafuertes/alminar }\end{array}$ & 14,41 & $\begin{array}{c}24,45= \\
24,50\end{array}$ & $30,56=30,50$ & 45,84 \\
\hline $\begin{array}{c}\text { Longitud pórtico sin } \\
\text { contrafuertes }\end{array}$ & 2,88 & 4,89 & 6,10 & 9,16 \\
\hline $\begin{array}{c}\text { Longitud pórtico con } \\
\text { contrafuertes }\end{array}$ & 4 & 6,78 & $8,48=8,50$ & 12,72 \\
\hline Radio círculo patio completo & 11,10 & 18,83 & $23,55=23,50$ & 35,31 \\
\hline Lado cuadrado inscrito & 15,70 & 26,64 & 33,30 & $49,95=50$ \\
\hline
\end{tabular}

Fig. 19: resumen de las dimensiones del patio de la mezquita menor de Madīnat al-Zahrā'.

MEZQUITA COMPLETA (Fig. 20): la equivalencia de estas dimensiones en pies no despeja ninguna de las incógnitas existentes. Los resultados obtenidos no nos han permitido determinar tampoco si longitud y anchura están relacionadas a través de alguna proporción concreta. Con todo, procederemos también a la contemplación de las dimensiones totales del inmueble.

\begin{tabular}{|c|c|c|c|c|}
\hline $\begin{array}{c}\text { MEZQUITA MENOR DE } \\
\text { MADİNAT AL-ZAHRA } \bar{A}^{\prime}\end{array}$ & Metros & $\begin{array}{c}\text { Codos } \\
\text { rassasís } \\
(0,5893)\end{array}$ & $\begin{array}{c}\text { Codos } \\
\text { mammunis } \\
(0,4714)\end{array}$ & $\begin{array}{c}\text { Pies } \\
(0,3143)\end{array}$ \\
\hline $\begin{array}{c}\text { Anchura exterior sin } \\
\text { contrafuertes }\end{array}$ & 15,54 & 26,37 & $32,96=33$ & $\begin{array}{c}49,44= \\
49,50\end{array}$ \\
\hline $\begin{array}{c}\text { Anchura exterior con } \\
\text { contrafuertes }\end{array}$ & 17,41 & $\begin{array}{c}29,54= \\
29,50\end{array}$ & $36,93=37$ & $\begin{array}{c}29,54= \\
29,50\end{array}$ \\
\hline $\begin{array}{c}\text { Longitud exterior sin } \\
\text { pórtico }\end{array}$ & 22,13 & $\begin{array}{c}37,55= \\
37,50\end{array}$ & $46,94=47$ & $\begin{array}{c}70,41= \\
70,50\end{array}$ \\
\hline $\begin{array}{c}\text { Longitud exterior sin } \\
\text { pórtico con contrafuertes }\end{array}$ & 23,31 & $\begin{array}{c}39,55= \\
39,50\end{array}$ & $49,45=49,50$ & 74,16 \\
\hline Longitud exterior & 25,02 & $\begin{array}{c}42,46= \\
42,50\end{array}$ & $53,07=53$ & 79,60 \\
\hline $\begin{array}{c}\text { Longitud exterior con } \\
\text { contrafuertes }\end{array}$ & 27,29 & 46,31 & 57,89 & 86,83 \\
\hline
\end{tabular}

Fig. 20: dimensiones totales de la mezquita menor de Madīnat alZahrā'.
La consideración de las medidas globales de la mezquita nos ha permitido encontrar, por fin, una proporción. La anchura exterior sin contrafuertes $(49,50$ pies) multiplicada por $\sqrt{ } 2$, da como resultado 70 pies exactos, que se aproximan considerablemente a los 70,50 pies que tiene la longitud exterior sin pórtico. Esto resulta mucho más relevante si recurrimos a la comparación de la metrología de esta mezquita con la de su aljama correspondiente.

Según A. Jiménez, la aljama de Madīnat al-Zahrā' "se trazó siguiendo la tradición geométrica iniciada en Damasco" (Jiménez Hernández 2015: 20), comenzándose su diseño, una vez más, a partir del haram. La longitud interior del mismo equivalió a 76 pies, cuya multiplicación por $\sqrt{2}$ habría dado la anchura de dicho espacio, esto es, 107,5 pies. El patio, por su parte, tuvo esa misma anchura, además de una longitud de 87,5 pies. Teniendo en cuenta también la propuesta de A. Jiménez de que el haram de esta aljama se planteó desde adentro hacia afuera porque primó el cálculo de la capacidad que se deseaba que albergase, hemos aplicado dicha hipótesis a esta mezquita menor (Fig. 21).

\begin{tabular}{|c|c|c|c|c|}
\hline $\begin{array}{c}\text { MEZQUITA MENOR DE } \\
\text { MADINAT AL-ZAHRA' } \\
\text { SALA DE ORACION } \\
\text { (INTERIOR) }\end{array}$ & Metros & $\begin{array}{c}\text { Codos } \\
\text { rassasis } \\
(0,5893)\end{array}$ & $\begin{array}{c}\text { Codos } \\
\text { mammunis } \\
(0,4714)\end{array}$ & $\begin{array}{c}\text { Pies } \\
(0,3143)\end{array}$ \\
\hline Anchura interior & 13,97 & 23,70 & 29,63 & $\begin{array}{c}44,45= \\
44,50\end{array}$ \\
\hline Longitud interior & 9,89 & 16,78 & $20,98=21$ & $\begin{array}{c}31,47= \\
31,50\end{array}$ \\
\hline
\end{tabular}

Fig. 21: dimensiones interiores de la sala de oración de la mezquita menor de Madīnat al-Zahrā'.

Obtenemos así que no sólo las medidas lineales son enteras en pies, sino que la longitud multiplicada por $\sqrt{2}$ efectivamente resulta en la anchura del oratorio: 31,5 $x \sqrt{2}=44,54$. Con todo, debemos ser cautos, pues nos encontramos ante una proporción que sólo hemos sido capaces de rastrear en este caso, y que dejamos aquí apuntada para tener en cuenta en un futuro, si apareciesen más ejemplos rectangulares susceptibles de ser analizados a través de esta metodología.

\section{Caso 4: mezquita de Santa Clara}

Este es, quizás, el caso más complicado de cuantos venimos analizando hasta ahora pues, pese al estado de conservación excepcional del conjunto, lo cierto es que hay detalles importantes que aún se desconocen. Paradójicamente, las numerosas excavaciones acontecidas en su 
seno no siempre han conducido a una mejor comprensión de la evolución y la cronología del inmueble, del cual ni siquiera se había ofrecido, hasta la fecha (González Gutiérrez 2016a: 179 y ss.), una planta de la fase islámica que completase o corrigiese las hipótesis de F. Hernández (1975: 204, fig. 43) o V. Escribano (1964-1965: 89). Las dimensiones consignadas por los distintos investigadores que han intervenido en el edificio no siempre coinciden, por lo que resulta arduo, cuando no imposible, obtener tablas de medidas rigurosas (Ibid.: 182 y ss; tablas $10 \mathrm{y}$ 11). Por otra parte, las dificultades que entraña el acceso a su interior para un reconocimiento in situ, así como su estado semi ruinoso, nos han impedido llevar a cabo mediciones adecuadas o lo suficientemente precisas para el análisis que proponemos.

A pesar de las citadas adversidades, la conjugación entre los datos recogidos por todas las intervenciones previas y los tomados por nosotros, nos permiten ofrecer una planta provisional de la antigua mezquita que, si bien resulta parcialmente hipotética, se convierte en una primera base sobre la que trabajar en esta línea.

SALA DE ORACIÓN: Dadas las similitudes que el edificio guarda con la mezquita de Fontanar, podemos sugerir que su planteamiento constructivo fue similar. Nos encontramos ante una sala de oración de planta cuadrada, por lo que cabe pensar que ésta se trazó, de nuevo, a partir de un círculo (Fig. 22). Consideramos las dimensiones exteriores, condicionadas por la existencia de cuatro contrafuertes a cada lado de la sala.

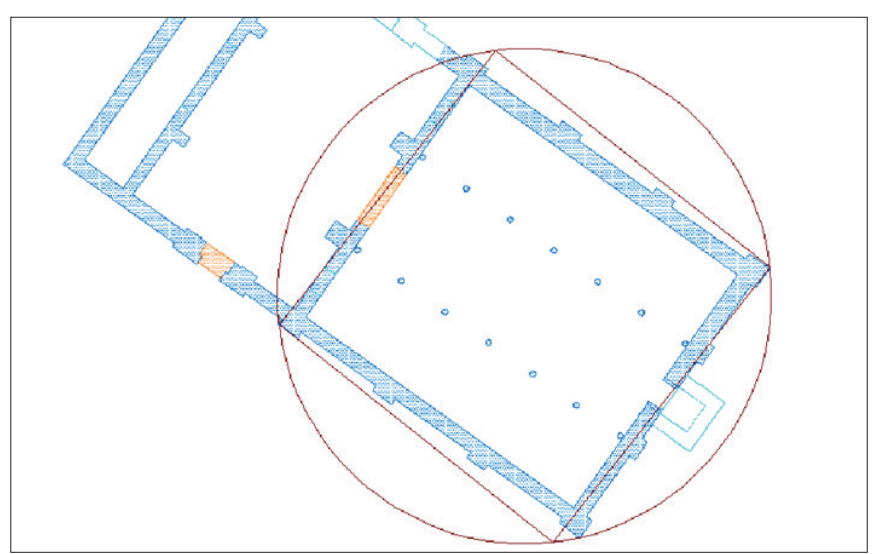

Fig. 22: hipótesis de la obtención de la sala de oración de la mezquita de Santa Clara a partir de un círculo.

\begin{tabular}{|c|c|c|c|c|}
\hline $\begin{array}{c}\text { SANTA CLARA: } \\
\text { SALA DE } \\
\text { ORACIÓN }\end{array}$ & Metros & $\begin{array}{c}\text { Codos rassasis } \\
(0,5893)\end{array}$ & $\begin{array}{c}\text { Codos } \\
\text { mammunis } \\
(0,4714)\end{array}$ & $\begin{array}{c}\text { Pies } \\
(0,3143)\end{array}$ \\
\hline Anchura exterior & 17,7 & $30,03=30$ & $37,55=37,50$ & 56,31 \\
\hline Longitud exterior & 19,71 & $33,45=33,50$ & 31,80 & 62,71 \\
\hline Radio círculo & 12,57 & 21,33 & 26,66 & 40 \\
\hline $\begin{array}{c}\text { Lado del cuadrado } \\
\text { inscrito }\end{array}$ & 17,77 & 30,15 & 37,69 & $\begin{array}{c}56,53= \\
56,50\end{array}$ \\
\hline $\begin{array}{c}\text { Diagonal del } \\
\text { cuadrado inscrito }\end{array}$ & 25,14 & 42,66 & 53,33 & $79,98=80$ \\
\hline
\end{tabular}

Fig. 23: dimensiones del haram de la mezquita de Santa Clara.

A partir de la figura 23 podemos lanzar las siguientes deducciones: parece que, en efecto, el arquitecto pudo esbozar este haram según el sistema que ya hemos comentado, a partir de un círculo con un radio de 40 pies en el que se inscribió un cuadrado de 56,5 pies de lado. No obstante, quizás la ejecución de la obra no pudiese ajustarse con exactitud a dicho planteamiento ya que, pese a que la anchura real de la sala tuvo finalmente 56,31 pies -que sí se aproximan a los 56,5 planteados-, la longitud excedió bastante esa cantidad. Esta es una hipótesis más que razonable si tenemos en cuenta que, por primera vez en todos los casos analizados, no estamos ante una construcción que dispusiese de un terreno libre de edificaciones, sino que hubo de ajustarse a un solar muy específico que, además, también contaba con estructuras previas (Ruiz Bueno 2016: 410-418; 670671). De la misma manera, el urbanismo ya existente del entorno debió de influir en la puesta en marcha del edificio que nos ocupa. En otro orden de cosas, cabe asimismo la posibilidad de que las inexactitudes que pueda albergar nuestro plano influyan en esta distorsión.

PATIO: la aplicación de la misma metodología que estamos considerando para los casos anteriores resulta así:

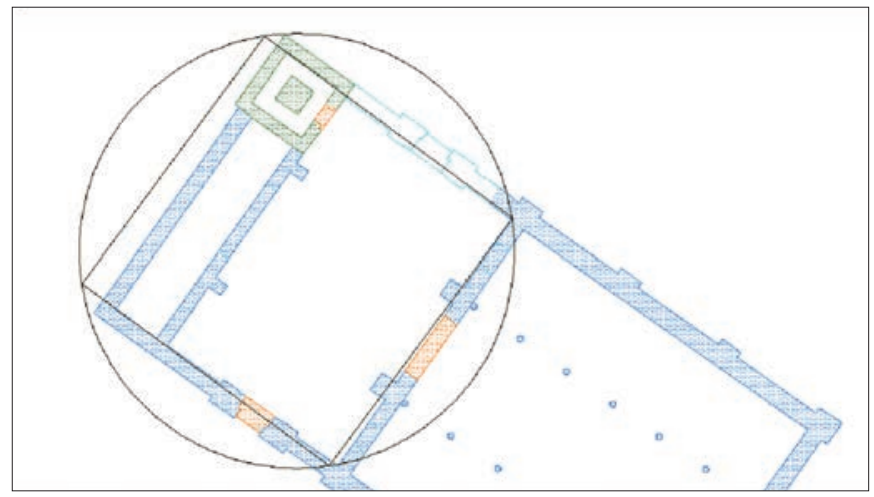

Fig. 24: hipótesis del trazado del patio de la mezquita de Santa Clara a partir de un círculo. 


\begin{tabular}{|c|c|c|c|c|}
\hline $\begin{array}{c}\text { SANTA CLARA: } \\
\text { PATIO }\end{array}$ & Metros & $\begin{array}{c}\text { Codos } \\
\text { rassasís } \\
(0,5893)\end{array}$ & $\begin{array}{c}\text { Codos } \\
\text { mammunis } \\
(0,4714)\end{array}$ & $\begin{array}{c}\text { Pies } \\
(0,3143)\end{array}$ \\
\hline Anchura exterior & 17,7 & $30,03=30$ & $37,55=37,50$ & 56,31 \\
\hline $\begin{array}{c}\text { Longitud exterior sin } \\
\text { pórtico }\end{array}$ & 11,14 & 18,90 & 23,63 & $\begin{array}{c}35,44= \\
35,50\end{array}$ \\
\hline Longitud pórtico & 3,35 & 5,68 & 7,10 & 10,65 \\
\hline $\begin{array}{c}\text { Longitud patio } \\
\text { completo }\end{array}$ & 14,50 & 24,60 & 30,76 & 46,13 \\
\hline $\begin{array}{c}\text { Radio círculo con } \\
\text { pórtico }\end{array}$ & 10,97 & 18,61 & 23,27 & 34,90 \\
\hline $\begin{array}{c}\text { Lado del cuadrado } \\
\text { inscrito }\end{array}$ & 15,51 & 26,32 & 32,90 & 49,35 \\
\hline $\begin{array}{c}\text { Diagonal del } \\
\text { cuadrado inscrito }\end{array}$ & 21,94 & 37,23 & $46,54=46,50$ & 69,80 \\
\hline
\end{tabular}

Fig. 25: dimensiones del patio de la mezquita de Santa Clara.

Aunque, al igual que ocurre en los ejemplos precedentes, el patio parece sufrir más desajustes que el haram, en este caso el descuadre se dispara, ya que apenas ninguna medida parece haber sido tomada en pies enteros, ni en la hipótesis de plano ni en la obra final. Al margen de que los posibles errores contenidos en nuestra propuesta planimétrica puedan influir en esta cuestión, podría haber ocurrido que esta mezquita experimentase distintas fases constructivas. Estas, además de evidenciarse quizás en las diferencias de aparejo observadas al exterior (González Gutiérrez 2016a: 188), anularían el sentido de un análisis global del patio.

MEZQUITA COMPLETA: dada la utilidad que ha demostrado tener para el resto de ejemplos contemplados en nuestra propuesta, hemos llevado a cabo, asimismo, el análisis del edificio en su conjunto (Fig. 26).

\begin{tabular}{|c|c|c|c|c|}
\hline $\begin{array}{c}\text { SANTA CLARA: } \\
\text { MEZQUITA } \\
\text { COMPLETA }\end{array}$ & Metros & $\begin{array}{c}\text { Codos } \\
\text { rassasis } \\
(0,5893)\end{array}$ & $\begin{array}{c}\text { Codos } \\
\text { mammmumis } \\
(0,4714)\end{array}$ & Pies (0,3143) \\
\hline Anchura exterior & 17,7 & $30,03=30$ & $37,55=37,50$ & 56,31 \\
\hline $\begin{array}{c}\text { Longitud exterior } \\
\text { sin pórtico }\end{array}$ & 30,64 & $51,99-52$ & 65 & $97,49=97,50$ \\
\hline Longitud total & 34 & 57,69 & 72,12 & 108,17 \\
\hline
\end{tabular}

Fig. 26: medidas de la mezquita de Santa Clara completa.

Según estos datos, podemos plantear que la construcción de esta mezquita se llevó a cabo partiendo primero del diseño de su sala de oración. Ésta se proyectó desde el exterior hasta el interior, y no a la inversa, por las limitaciones de espacio que comentábamos más arriba, lo cual significaría que el arquitecto artífice del proyecto hizo uso del solar del que disponía desde fuera hacia dentro. Así, pudo trazar las líneas fundamentales de la estructura del haram, y a partir de éstas diseñó el resto del inmueble.

\section{RESULTADOS PRELIMINARES Y REFLEXIONES PARA FUTURAS INTERVENCIONES}

Tras este recorrido, creemos posible afirmar que los espacios religiosos analizados se plantearon y edificaron siguiendo el pie como unidad de medida. Este estudio contribuye así, de alguna manera, a desterrar la creencia de que el sistema de medidas medieval islámico era caótico, no reglado o incluso aleatorio. La construcción de estas mezquitas partió del diseño de su haram, si bien no hemos sido capaces de hallar una proporción general que justifique la elección de unas u otras dimensiones, o que determine el tamaño del patio en función del de la sala de oraciones. Parece que estos diseños se llevaron a cabo desde fuera hacia dentro, aunque en el caso 2 (Ronda Oeste), es posible que el planeamiento se realizase a la inversa, primándose el cálculo del aforo deseado para este oratorio.

El análisis de las mezquitas pertenecientes a Qurțuba nos sugiere la existencia de un posible esquema de construcción de las mezquitas califales (en este análisis, Fontanar y Santa Clara), conformadas por una sala de oración de planta cuadrada y, a continuación, un patio porticado en su lado noroeste que parece tener también una forma cuadrangular de igual superficie, o ligeramente mayor, a la de la sala de oración (Fig. 27). Este tipo de mezquita reproduce, a grandes rasgos, el esquema de doble cuadrado de la aljama cordobesa en época califal. Por su parte, el análisis del conjunto aparecido en la Ronda Oeste no nos ha permitido despejar contundentemente las incógnitas que albergábamos al inicio. Salta a la vista que esta construcción no encaja con el tipo de doble cuadrado recién descrito, lo cual puede llevarnos a pensar en una fundación emiral del conjunto. Además, la extraña disposición de su pórtico, detrás del alminar y no entregándose a un lateral del mismo, también nos lleva a pensar que se añadió en un momento posterior, cuando la mezquita y su alminar ya estaban construidos.

La aplicación de un modelo de doble cuadrado para las mezquitas califales de Córdoba no es, en principio, extrapolable a las demás mudun. En la vecina Madīnat al-Zahrā', por ejemplo, parece estar sucediendo algo bien distinto. La pequeña mezquita analizada más arriba (caso 3), aunque califal, se aleja del modelo de doble cuadrado para acercarse a las formas y proporciones de 


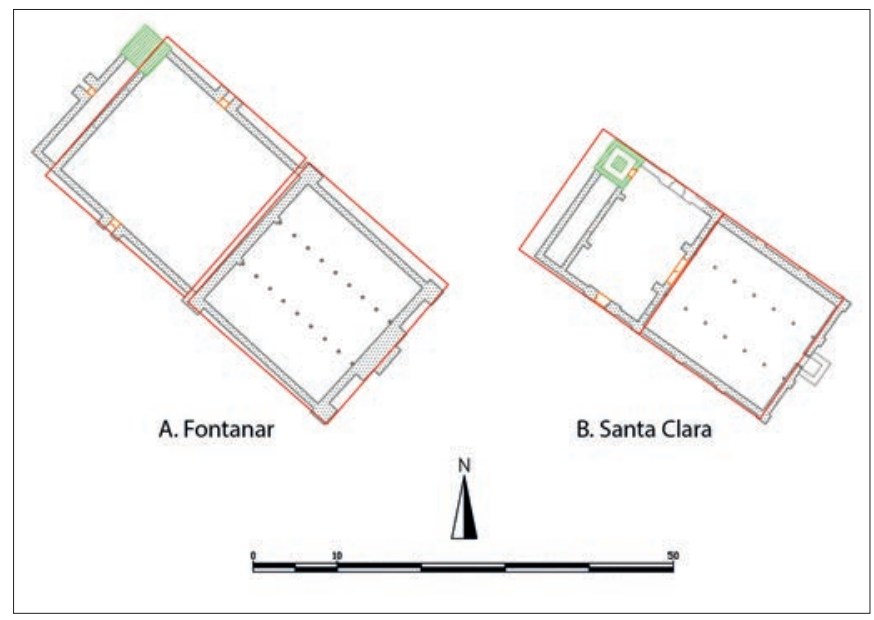

Fig. 27: mezquitas califales de Madīnat Qurțuba analizadas. A la izda., Fontanar. A la dcha., Santa Clara. Ambas responden a un modelo de doble cuadrado, con ligeras variaciones. En el primer caso, tanto la sala de oraciones como el patio sin su pórtico compartieron una superficie prácticamente idéntica. En el segundo, el pórtico se incluye en el cuadrado en el que se circunscribió el patio completo. Dicho cuadrado tuvo también unas dimensiones muy similares a las de la sala de oraciones.

la aljama de esta misma ciudad. Su sala de oraciones se concibió, otra vez, desde dentro hacia fuera, primándose la obtención de un aforo concreto. En este caso sí se ha podido comprobar la aplicación de una proporción específica, consistente en la multiplicación de la anchura del edificio por $\sqrt{ } 2$ para la obtención de su longitud total. Lamentablemente, estos resultados tampoco pueden trasladarse a otros ejemplos de este mismo asentamiento, puesto que sólo contamos con un caso analizado y, por tanto, insuficiente para derivar de él estadísticas o tendencias.

Sin embargo, sí creemos interesante implantar, a modo de hipótesis, el esquema califal de doble cuadrado que se ha podido identificar para Fontanar y Santa Clara (Fig. 27) a otros restos de mezquitas cordobesas que no han sido documentados en su totalidad. Nos referimos, entre otros, a los vestigios parciales que se encontraron en los terrenos de la actual estación de autobuses (vid. González Gutiérrez 2016a: 123 y ss.), muy precarios pero que permiten conocer cuál fue el perímetro aproximado del edificio, aunque sin detalles sobre su estructuración interna. Dicho perímetro dibuja una forma rectangular de, aproximadamente, $26,30 \mathrm{~m}$ de largo $\mathrm{x}$ 11,96 m. Hemos insertado en él dos formas cuadradas de 11,96 m de lado, una a modo de sala de oraciones con el mihrab al exterior, tal y como se ha considerado en los casos anteriores- y otra simulando el patio. El resultado obtenido nos sirve como propuesta preliminar de las formas de esta mezquita (Fig. 28).

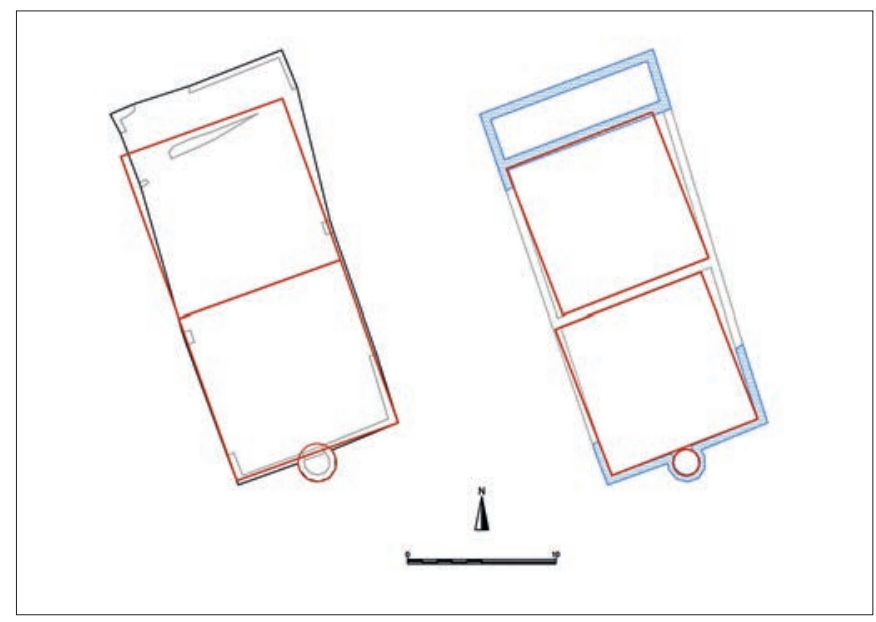

Fig. 28: superposición del esquema de doble cuadrado a los restos documentados en la estación de autobuses. A la izda., perímetro documentado para el edificio en el proceso de excavación (González Gutiérrez 2012: 144, fig. 12). A la dcha., una nueva propuesta planimétrica, en la que añadimos un muro de separación entre la sala de oración y el patio - del mismo espesor que los muros documentados-, junto con un pórtico en el extremo NW (González Gutiérrez 2016b: 278, fig. 4).

Este mismo procedimiento lo hemos trasladado a los restos documentados durante la excavación de las denominadas "Naves de Fontanar" (vid. González Gutiérrez 2016a: 243 y ss.), cuya planta se conoce con mayor exactitud que la del caso anterior, aunque se carece de estratigrafía asociada a la misma. ${ }^{13}$ Se trata de un edificio califal estructurado en dos cuerpos diferentes: un primer espacio compuesto por tres naves separadas por columnas, que podría ser interpretado como haram; y un posible sahn, también de planta cuadrada, con un espacio adosado a su lado noroeste que pudo constituir un pórtico. La superposición del esquema de doble cuadrado -en este caso de 11,49 m de lado-a la planimetría de este conjunto (Fig. 29) encaja casi a la perfección con la hipótesis que defendemos:

De esta manera, la sala de oración dibujaría una planta cuadrada, y el patio tendría aproximadamente las mismas dimensiones que ésta, o ligeramente mayores si incluimos el muro de separación entre esta zona y la anterior. El espacio sobrante correspondería al pórtico que, además, coincide con un pequeño espacio delimitado por un muro, detectado durante los trabajos de documentación del conjunto. La coincidencia casi exacta entre las áreas documentadas y el esquema hipotético que proponemos proporciona otro argumento sólido

\footnotetext{
13 El edificio ha sido prospectado en superficie, pero no excavado, por lo que no contamos con su secuencia estratigráfica ni con otros datos arqueológicos (vid. Murillo et al. 2004).
} 


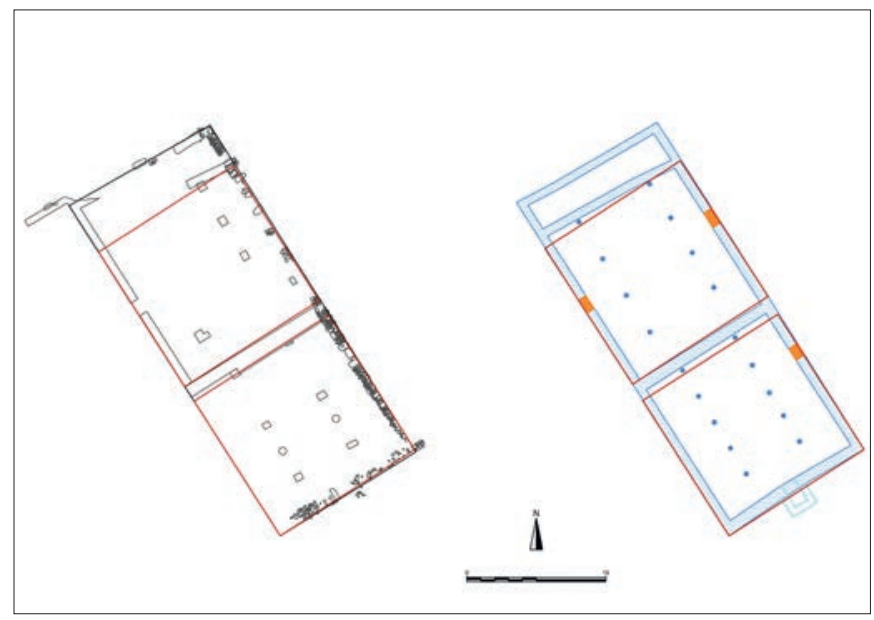

Fig. 29: superposición del esquema de doble cuadrado a los restos documentados de Naves de Fontanar. A la izda., planta documentada del edificio (González Gutiérrez 2012: 194, fig. 69). A la dcha., una nueva propuesta en la que diferenciamos su sala de oración y su patio, con un pórtico en el extremo NW (González Gutiérrez 2016b: 278, fig. 5).

a favor de la interpretación de este edificio como una mezquita, insegura según sus excavadores (Murillo et al. 2004: VII, 166). La aplicación de esta tipología de doble cuadrado a los casos dudosos resulta, a la luz de este ejemplo, bastante esclarecedora para ayudar en la identificación de posibles mezquitas en lo sucesivo, así como para afinar en cuestiones de cronología.

En cuanto a la correspondencia de estos dos últimos trazados esquemáticos con los pies islámicos, obtenemos, una vez más, la confirmación de la utilización de dicha unidad de medida:

- Para los restos sitos en la estación de autobuses, los $11,96 \mathrm{~m}$ de lado del cuadrado equivalen $38,05=38$ pies. Éste se habría inscrito en un círculo de $8,46 \mathrm{~m}$ de radio, es decir, 26,92 $=27$ pies.

- Para las Naves de Fontanar, el cuadrado propuesto de 11,49 $\mathrm{m}$ de lado se corresponde con 36,55 = 36,5 pies. Se inscribiría, a su vez, en un círculo cuyo radio habría sido de $8,125 \mathrm{~m}=25,85$ pies.

Desafortunadamente, carecemos de un número significativo de mezquitas emirales, por lo que no podemos proponer una cronotipología más amplia que rebase los límites del Califato. Aun así, ya hemos visto cómo el análisis métrico de la mezquita de la Ronda Oeste (caso 2), cuya cronología inicial ha sido objeto de intenso debate, ha suscitado la aparición de nuevos argumentos a favor de su origen emiral, así como de una posible ampliación de su patio durante el Califato.
Para terminar, si bien el escaso número de ejemplos con el que nos hemos visto obligados a trabajar ha limitado nuestro rango de actuación, la aplicación de esta metodología nos ha permitido entrever algunas líneas muy interesantes sobre las que seguir trabajando a la luz de nuevos ejemplos. Aunque, por el momento, no pueden erigirse como modelos generales, estos resultados pueden constituir pautas sobre las que seguir avanzando, o bien referencias para emprender estudios similares en otras ciudades andalusíes. Si bien algunas de estas hipótesis podrían confirmarse en el futuro mediante nuevos análisis y hallazgos arqueológicos, debemos subrayar que este tipo de análisis debería ser siempre complementario al registro estratigráfico y estar subordinado a la información vertida por el mismo.

\section{AGRADECIMIENTOS}

La propuesta de análisis recién presentada ha sido supervisada por los Profes. Dres. Desiderio Vaquerizo y Alberto León, a quienes agradecemos encarecidamente su dedicación y sus comentarios. Hacemos también extensible este agradecimiento al Dr. Alejandro Jiménez Hernández, quien ha tenido la amabilidad y el interés de orientarnos en todo momento a lo largo de este proceso de estudio, y con quien hemos tenido oportunidad de discutir y comentar en numerosas ocasiones los resultados que progresivamente íbamos obteniendo. Este trabajo es, sin duda, deudor de su labor, así como de sus indicaciones y apreciaciones. Por último, queremos mostrar nuestra enorme gratitud hacia D. José María Tamajón Navarro, cuya labor ha sido fundamental en el proceso de dibujo, corrección y reelaboración de los planos sobre los que se sustenta esta investigación.

\section{BIBLIOGRAFÍA}

Arnold, F. 2009-2010. "El edificio singular del Vial Norte del Plan Parcial Renfe. Estudio arquitectónico", Anejos de Anales de Arqueología Cordobesa, 2, pp. 247-274.

Camps, E. 1953. Módulo, proporciones y composición en la arquitectura califal cordobesa. CSIC, Madrid.

De la Hoz, R. 1973. La proporción cordobesa. Diputación Provincial, Córdoba. Escribano, V. 1964-1965. "La mezquita de la calle Rey Heredia", al-Mulk, 4, pp. 83-101.

Fernández Puertas, A. 2000. "Mezquita de Córdoba. Trazado proporcional de su planta general (siglos VIII-X)", Archivo Español de Arte, 291, pp. 217 247, doi: https://doi.org/10.3989/aearte.2000.v73.i291.829.

Fernández Puertas, A. 2008. "Mezquita de Córdoba: Abd al-Rahman I (169/785-786). El trazado proporcional de la planta y alzado de las arquerías del oratorio. La quibla y el mihrab del siglo VIII", Archivo Español 
de Arte, 324, pp. 333-356, doi: https://doi.org/10.3989/aearte.2008.v81. i324.127.

Fernández Puertas, A. 2009. "Mezquita de Córdoba: el trazado de la portada interior de la Bab al-Wuzara'. La Puerta de los Deanes (s. VIII), su trazado interior y exterior", Archivo Español de Arte, 326, pp. 107-136, doi: https://doi.org/10.3989/aearte.2009.v82.i326.146.

González Gutiérrez, C. 2016a. Las mezquitas de la Córdoba islámica: concepto, tipología y función urbana. Tesis Doctoral, Universidad de Córdoba.

González Gutiérrez, C. 2016b. "Las mezquitas de barrio de Madinat Qurtuba 15 años después: espacios religiosos urbanos en la capital andalusí", Anales de Arqueología Cordobesa, 27, pp. 267-292.

Hernández, F. 1961-1962. "El codo en la historiografía árabe de la mezquita mayor de Córdoba: contribución al estudio del monumento", al-Mulk, 2, pp. 5-52.

Hernández, F. 1975. El alminar de Abd al-Rahman III en la Mezquita Mayor de Córdoba: génesis y repercusiones. Patronato de la Alhambra, Granada. Jiménez Hernández, A. 2015. "La metrología histórica como herramienta para la Arqueología de la Arquitectura. La experiencia en los Reales Alcázares de Sevilla", Arqueología de la Arquitectura 12, pp. 1-29, doi: https:// doi.org/10.3989/arq.arqt.2015.001.
Luna, D. y Zamorano, A. 1999. "La mezquita de la antigua finca El Fontanar (Córdoba)", Cuadernos de Madinat al-Zahra, 4, pp. 145-173.

Murillo, J. F.; Bermúdez, J. M.; Barbero, I; Castro, E.; Pizarro, G.; Rodríguez, M. C.; Salinas, J. M. y Sánchez, I. 2004. Intervención Arqueológica de Urgencia. Edificio de Usos Múltiples del Área de Infraestructuras del Ayuntamiento de Córdoba. El Fontanar, Parque Cruz Conde (Córdoba), vols. I, III, IV, VI y VII, Córdoba, Inédito.

Murillo, J. F.; Casal, M. T. y Castro, E. 2004. "Madinat Qurtuba. Aproximación al proceso de formación de la ciudad emiral y califal a partir de la información arqueológica", Cuadernos de Madinat al-Zahra, 5, pp. 257- 290.

Roldán, F. J. 2013. Principios de metrología en la arquitectura del pasado. Tesis Doctoral, Universidad de Granada.

Ruiz Bueno, M. D. 2016. Topografia, imagen y evolución urbanística de la Córdoba clásica a la tardoantigua (ss. II-VII d. C.). Tesis Doctoral, Universidad de Córdoba.

Vallejo, A. 2009. "Intervención arqueológica en el tramo sur de la muralla de Madīnat al-Zahrā' 2007-2008”, Patrimonio cultural de España, 0, pp. 215-224.

Vallvé, J. 1976. "Notas de metrología hispano-árabe. El codo en la España musulmana", al-Andalus, 41-II, pp. 339-354. 